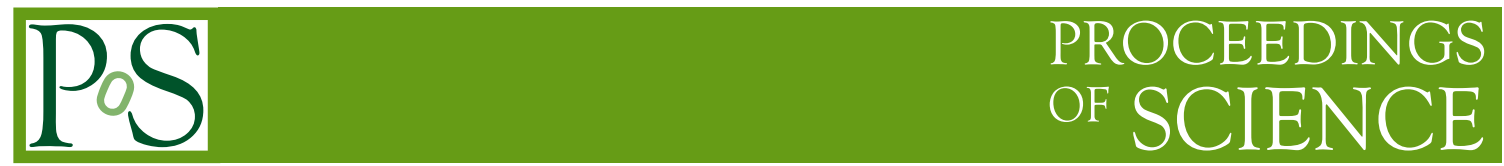

\title{
Scattering Amplitudes in Three Dimensions
}

\author{
Arthur Lipstein* \\ $\dagger$ \\ The Mathematical Institute, University of Oxford, \\ 29-29 St Giles', Oxford, OXI 3LB, UK \\ E-mail: Arthur.Lipstein@maths.ox.ac.uk
}

In this note, we review some recent progress in understanding planar, on-shell, color-ordered scattering amplitudes of $3 \mathrm{~d}$ gauge theories. In particular, we will discuss the amplitudes of $3 \mathrm{~d}$ Yang-Mills theory with maximal supersymmetry as well as mass-deformed $3 \mathrm{~d}$ gauge theories with $\mathscr{N}=2$ supersymmetry. This note is primarily based on [12] and [13].

Proceedings of the Corfu Summer Institute 2012

September 8-27, 2012

Corfu, Greece

\footnotetext{
* Speaker.

$\dagger$ I would like to thank my collaborators on the papers on which this note is based. In particular, I thank Lionel Mason for collaborating with me on [12] and I would like to thank Abhishek Agarwal and Donovan Young for collaborating with me on [13].
} 


\section{Introduction}

Over the past few years, there has been a great deal of progress in understanding the scattering amplitudes of three-dimensional gauge theories. In particuar, a BCFW recursion relation for threedimensional gauge theories with massless fields was developed, and used to show that an $\mathscr{N}=6$ superconformal Chern-Simons theory known as the ABJM theory [2] has dual superconformal symmetry $[1,3,4]$. Furthermore, some evidence for an amplitude/Wilson loop duality in the ABJM theory was found $[5,6,7]$, and it was shown that the amplitudes of the ABJM theory can be be encoded in an integral over the orthogonal Grassmannian [8]. Recently, 1-loop amplitudes were computed in the ABJM theory and shown to exhibit new structures such as sign functions of the kinematic variables $[9,10,11]$.

More recently, progress has been made in understanding the amplitudes of maximal $3 \mathrm{~d}$ superYang-Mills theory [12] and mass-deformed 3d gauge theories with $\mathscr{N}=2$ supersymmetry, notably mass-deformed Chern-Simons theory and Yang-Mills-Chern-Simons theory [13]. As we will describe in greater detail, the amplitudes of these theories exhibit many surprising properties. For example, the amplitudes of $\mathscr{N}=8$ super-Yang-Mills have enhaced R-symmetry [14], dual conformal covariance, and loop structure which resembles that of the ABJM theory [15]. Furthermore, the four-point amplitudes of mass-deformed $\mathscr{N}=2$ Chern-Simons theory can be encoded in a very simple superamplitude. In $\mathscr{N}=2$ Yang-Mills-Chern-Simons theory, it is possible to compute amplitudes without external gauge fields using Feynman diagram techniques and to deduce the remaining amplitudes using supersymmetry constraints, at least at four-points.

In section 2, we will describe the amplitudes of $\mathscr{N}=8$ super-Yang-Mills theory. In particular, we will explain how they can be obtained by dimensional reduction of $4 \mathrm{~d} \mathscr{N}=4$ super-Yang-Mills theory. We also explain how dual conformal covariance is realized in these amplitudes, and briefly describe the structure of loop amplitudes in $\mathscr{N}=8$ super-Yang-Mills theory. In section 3, we describe the amplitudes of mass-deformed $3 \mathrm{~d}$ gauge theories with $\mathscr{N}=2$ supersymmetry. We also describe a proposal for BCFW recursion relations for massive $3 \mathrm{~d}$ gauge theories. In section 4 , we present some conclusions and open questions.

\section{3d Yang-Mills Theory}

In this section, we will describe the scattering amplitudes of maximal 3d super Yang-Mills theory. In addition to having a Yang-Mills gauge field with gauge group $U(N)$, it has seven scalars and eight fermionic degrees of freedom which are in the adjoint of the gauge group. This theory can be obtained by dimensional reduction of maximal 4d super Yang-Mills theory [16]. Note that $3 \mathrm{~d}$ Yang-Mills theory is not conformal because the Yang-Mills coupling is dimensionful. Nevertheless, the amplitudes of maximal 3d super Yang-Mills theory have a property known as dual conformal covariance, which cannot be seen from the spacetime Lagrangian. We will describe this in greater detail in the following subsections.

\subsection{Dimensional Reduction}

The on-shell scattering amplitudes of $3 \mathrm{~d}$ Yang-Mills theories can be obtained by dimensional reduction of $4 \mathrm{~d}$ Yang-Mills amplitudes. To see how this is achieved, consider a null $4 \mathrm{~d}$ null mo- 
mentum written in bispinor form:

$$
p^{\alpha \dot{\beta}}=\lambda^{\alpha} \bar{\lambda}^{\dot{\beta}}
$$

where $\alpha=1,2$ and $\dot{\beta}=1,2$ are $S U$ (2) indices which arise from the fact that the Lorentz group is locally $S O(4) \sim S U(2)_{L} \times S U(2)_{R}$. When reducing to three dimensions, the distinction between dotted and undotted indices disappears because the Lorentz group is $S U(2)=\left[S U(2)_{L} \times S U(2)_{R}\right]_{\text {diagonal }}$. Hence, to reduce to three dimensions, we simpy change dotted indices to undotted indices and symmetrize:

$$
p^{\alpha \beta}=\lambda^{\left(\alpha \bar{\lambda}^{\beta)}\right.} .
$$

Note that after symmetrizing, the momentum has three components, as expected. The magnitude of the three-momentum is

$$
p^{2}=\frac{1}{4}\langle\lambda \bar{\lambda}\rangle^{2}
$$

where $\left\langle\lambda_{i} \lambda_{j}\right\rangle=\varepsilon_{\alpha \beta} \lambda_{i}^{\alpha} \lambda_{j}^{\beta}$. If the three-momentum is null, then $\lambda \propto \bar{\lambda}$ and the momentum can be written in bispinor form as

$$
p^{\alpha \beta}=\lambda^{\alpha} \lambda^{\beta}
$$

In summary, if we wish to reduce the tree-level amplitudes of $4 \mathrm{~d}$ super-Yang-Mills to $3 \mathrm{~d}$ without breaking supersymmetry, we change dotted indices to undotted indices and leave fermionic coordinates untouched. Using this reduction procedure, it is easy to see that the MHV amplitudes of maximal $3 \mathrm{~d}$ super-Yang-Mills are the same as those of maximal $4 \mathrm{~d}$ super-Yang-Mills with a reduced supermomentum delta function:

$$
\mathscr{A}_{n}^{M H V}=\frac{\delta^{3}(P) \delta^{8}(Q)}{\langle 12\rangle\langle 23\rangle \ldots\langle n 1\rangle}
$$

where $n$ is the number of external legs, $P=\sum_{i=1}^{n} \lambda_{i}^{\alpha} \lambda_{i}^{\beta}, Q^{I \alpha}=\sum_{i=1}^{n} \lambda_{i}^{\alpha} \eta_{i}^{I}$, and $\delta^{8}(Q)=\Pi_{I=1}^{4} Q^{I \alpha} Q_{\alpha}^{I}$. Furthermore, $\eta_{i}^{I}$ is a fermionic variable where $I=1, \ldots, 4$ is an $S U(4)$ R-symmetry index and $i$ labels the external legs.

Note that $\mathscr{N}=8$ super-Yang-Mills has seven scalars and therefore has $S O(7)$ R-symmetry. On the other hand, only $S U(4)$ R-symmetry is manifest in the amplitudes obtained by dimensional reduction. This can be understood as follows. One can then define a $U(1) \mathrm{R}$-symmetry which rotates this scalar and another scalar in the theory, and encodes the helicity [17, 14]. Hence, in order to define helicity in $\mathscr{N}=8$ super-Yang-Mills, we must break the $S O(7)$ R-symmetry to $S O(6) \sim S U(4)$. It is possible to show that the 4-point superamplitude actually has $S O(8) \mathrm{R}$ symmetry [14], but this does not persist for higher point amplitudes. For $n>4$, there is a single $n$-point amplitude with $S O(7) \mathrm{R}$-symmetry, which can be constructed as a linear combination of $n$-point amplitudes with different MHV degrees.

\subsection{Dual Conformal Covariance and Loop Structure}

Although the amplitudes of $\mathscr{N}=8$ super-Yang-Mills can be obtained by dimensional reduction of $\mathscr{N}=4$ super-Yang-Mills amplitudes, they have many properties which do not follow trivially from dimensional reduction. For example, they have a property known as dual conformal covariance. To see this, consider arranging the external supermomenta of a scattering amplitude 
into a null polygon and expressing the amplitude as a function of the vertices of this polygon. In equations, the vertices of the polygon satisfy

$$
\left(x_{i}-x_{i+1}\right)^{\alpha \beta}=\lambda_{i}^{\alpha} \lambda_{i}^{\beta},\left(\theta_{i}-\theta_{i+1}\right)^{I \alpha}=\lambda_{i}^{\alpha} \eta_{i}^{I} .
$$

We will refer to $(x, \theta)$ as the dual space. Showing dual conformal covariance boils down to showing that the amplitudes transform covariantly under dual inversions:

$$
I\left[x^{\alpha \beta}\right]=\frac{x^{\alpha \beta}}{x^{2}}, I\left[\theta^{I \alpha}\right]=\frac{x^{\alpha \beta} \theta_{\beta}^{I}}{x^{2}} .
$$

If we strip the MHV amplitudes in eq 2.2 of the supermomentum delta function, it is not difficult to see that the remaining piece transforms covariantly under a dual inversion:

$$
I\left[\frac{1}{\langle 12\rangle\langle 23\rangle \ldots\langle n 1\rangle}\right]=x_{1}^{2} x_{2}^{2} \ldots x_{n}^{2} \frac{1}{\langle 12\rangle\langle 23\rangle \ldots\langle n 1\rangle} .
$$

More generally, using the $3 \mathrm{~d}$ BCFW recursion relation proposed in $[1]^{1}$, one can inductively show that if we decompose a general $n$-point amplitude as follows

$$
\mathscr{A}_{n}=\delta^{3}(P) \delta^{8}(Q) f_{n},
$$

then

$$
I\left[f_{n}\right]=x_{1}^{2} x_{2}^{2} \ldots x_{n}^{2} f_{n}
$$

Hence, although maximal 3d super-Yang-Mills theory does not have ordinary conformal symmetry (since the Yang-Mills coupling is dimensionful), it has dual conformal symmetry at tree-level.

Using unitarity, one can then show that the cut-constructable integrand of an $n$-point amplitude with $L$ loops transforms as follows under a dual inversion:

$$
I\left[\mathscr{I}_{n}^{L}\right]=\Pi_{i=1}^{n} x_{i}^{2} \Pi_{j=1}^{L}\left(x_{j}^{2}\right)^{4} \mathscr{I}_{n}^{L}
$$

where $i$ runs over the external regions and $j$ runs over loop regions in the dual space. In $D$ dimensions, the loop integration measure will give an additional factor of $\Pi_{j=1}^{L}\left(x_{j}^{2}\right)^{-D}$ under dual inversion. Hence, we find that the cut-constructable loop integrands transform like $4 \mathrm{~d}$ loop integrands under a dual inversion, i.e. the loop amplitudes have dual conformal symmetry if the loop momenta are taken to be four dimensional. This implies that the loop amplitudes of $3 \mathrm{~d} \mathscr{N}=8$ super-YangMills can be obtained by restricting $4 \mathrm{~d} \mathscr{N}=4$ super-Yang-Mills amplitudes to $3 \mathrm{~d}$ kinematics. In particular, to obtain a loop amplitude in $3 \mathrm{~d} \mathscr{N}=8$ super-Yang-Mills, one simply takes the corresponding $4 \mathrm{~d} \mathscr{N}=4$ super-Yang-Mills loop integrand and integrates over three-dimensional loop momenta.

The 1-loop amplitudes of $4 \mathrm{~d} \mathscr{N}=4$ super-Yang-Mills can be reduced to scalar box diagram [19]. In $D$-dimensions, this diagram is given by the following integral:

$$
I_{4}\left(p_{1}, p_{2}, p_{3}, p_{4}\right)=\int \frac{d^{D} l}{l^{2}\left(l+p_{1}\right)^{2}\left(l+p_{1}+p_{2}\right)^{2}\left(l+p_{1}+p_{2}+p_{3}\right)^{2}} .
$$

\footnotetext{
${ }^{1}$ Although 3-point amplitudes vanish on the support of their supermomentum delta functions when all the external momenta are null, one must include 3-point MHV and anti-MHV superamplitudes in the BCFW recursion relations of $\mathscr{N}=8$ super-Yang-Mills. For more details, see [12].
} 
Although the propagators in the box diagram are massless, the external legs can be massive. Setting $D=3+2 \varepsilon$ and expanding around $\varepsilon=0$, one finds the box diagram is finite if all four legs are massive, three legs are massive, or two adjacent legs are massive, and zero otherwise. This implies that in $\mathscr{N}=8$ super-Yang-Mills, the 1-loop correction vanishes for MHV amplitudes and is finite for non-MHV amplitudes. This is very similar to the structure of loop amplitudes in a $3 \mathrm{~d}$ superconformal Chern-Simons theory known as the ABJM theory, and suggests that there is a way to relate the amplitudes of $\mathscr{N}=8$ super-Yang-Mills and the ABJM theory order by order in perturbation theory. Indeed, the 2-loop 4-point amplitudes of both theories can be matched in the Regge-limit [15].

\section{Mass-Deformed Theories}

In this section, we describe the amplitudes of mass-deformed 3d gauge theories with $\mathscr{N}=2$ supersymmetry. Mass-deformed Chern-Simons theories with $\mathscr{N} \geq 4$ supersymmetry were first studied in [18]. The mass deformations we consider preserve locality, Lorentz invariance, and gauge invariance. In particular, there are two types of gauge theories in three dimensions with these properties, notably mass-deformed Chern-Simons theory and Yang-Mills-Chern-Simons theory. In the mass-deformed Chern-Simons theory, the gauge field is massless and does not have propagating degrees of freedom. Hence, only the matter fields can appear in the external legs of on-shell amplitudes and amplitudes with an odd number of external legs vanish on-shell (since these amplitudes have at least one external gauge field). In the Yang-Mills-Chern-Simons theory, the Chern-Simons term provides a topological mass for the gauge field, so the gauge field has one massive propagating degree of freedom [20,21]. As a result, both gauge and matter fields can appear in the exernal legs of on-shell amplitudes. For simplicity, we will take all the external legs to have mass $m$. In order to describe the amplitudes of these theories, it is convenient to express the momenta of external legs in bispinor form using eq 2.1. In particular, the spinors satisfy

$$
\langle\lambda \bar{\lambda}\rangle=2 m i
$$

\subsection{Mass-Deformed Chern-Simons Theory}

This theory has one $S U(N)$ gauge field, two massive scalars, and two massive Majorana fermions in the adjoint representation of the gauge group. In particular, it has $S O(2)$ R-symmetry which rotates the two scalars and two spinors, respectively. Furthermore, the supersymmetry algebra for this theory has a $U(1)$ extension:

$$
\left\{Q^{\alpha I}, Q^{\beta J}\right\}=\frac{1}{2}\left(P^{\alpha \beta} \delta^{I J}+m \varepsilon^{\alpha \beta} R^{I J}\right)
$$

where $I, J$ are the $S O(2)$ R-symmetry indices of the matter fields and $R^{I J}$ is the $S O(2)$ R-symmetry generator. The odd-point amplitudes vanish on-shell, so the first nontrivial amplitudes appear at four-points. Remarkably, all of the four-point amplitudes can be encoded in a single superamplitude:

$$
\mathscr{A}_{4}=\frac{\langle 42\rangle}{\langle\overline{3} 2\rangle} \delta^{3}(P) \delta^{2}(Q)
$$


where $P=\sum_{i=1}^{4} \lambda_{i}^{(\alpha} \bar{\lambda}_{i}^{\beta)}, Q^{\alpha}=\sum_{i=1}^{4}\left(\lambda_{i}^{\alpha} \bar{\eta}_{i}+\bar{\lambda}_{i}^{\alpha} \eta_{i}\right), \delta^{2}(Q)=Q^{\alpha} Q_{\alpha}$, and $\eta_{i}$ is a complex fermionic variable.

\subsection{Yang-Mills-Chern-Simons Theory}

This theory has one $S U(N)$ gauge field with both Yang-Mills and Chern-Simons terms. As we mentioned earlier, the Chern-Simons term gives the gauge field a topological mass, so the guage field has one massive degree of freedom. In addition, the theory has one massive scalar and two massive Majorana fermions in the adjoint of the gauge group. Note that there is $S O(2) \mathrm{R}$-symmetry in the fermionic sector but no R-symmetry in the bosonic sector. Unlike in the mass-deformed Chern-Simons theory, the supersymmetry algebra of the Yang-Mills-Chern-Simons theory has no $U(1)$ extension:

$$
\left\{Q^{\alpha I}, Q^{\beta J}\right\}=\frac{1}{2} P^{\alpha \beta} \delta^{I J}
$$

where $I, J$ are the $S O(2)$ R-symmetry indices of the fermions. The supersymmetry algebra can be used to derive constraints on amplitudes with more than three external legs.

There are various difficulties that arise when trying to compute Yang-Mills-Chern-Simons amplitudes with external gauge fields using Feynman diagrams. These complications stem from the difficulty in defining a mode expansion for the gauge field due to subtleties with canonical quantization. As a result, Feynman rules for external gauge fields in Yang-Mills-Chern-Simons theory are substantially more complicated than those of Yang-Mills theory. On the other hand, at least at four points in the $\mathscr{N}=2$ Yang-Mills-Chern-Simons theory, it is possible to compute all of the on-shell amplitudes without external gauge fields using Feynman diagrams and then deduce the remaining amplitudes using algebraic constraints arising from supersymmetry. Unlike in the mass-deformed Chern-Simons theory, it does not appear possible to encode the amplitudes of the $\mathscr{N}=2$ Yang-Mills-Chern-Simons theory in superamplitudes.

\subsection{BCFW}

A very useful technique for computing on-shell scattering amplitudes is the BCFW recursion relation [22]. For massless gauge theories in higher than three dimensions, these recursion relations are usually derived by linearly deforming two external momenta by complex parameter $z$ in such a way that momentum remains conserved and the momenta remain null. This deformation does not work in three dimensions, however. Rather, for massless $3 \mathrm{~d}$ gauge theories, one must deform two external momenta nonlinearly in the complex parameter $z$ (see [1] for more details). Furthermore, there is a natural way to generalize this deformation to massive $3 \mathrm{~d}$ gauge theories. In particular, consider two massive external legs $i, j$. The sum of the momenta of these legs is given by:

$$
\left(p_{i}+p_{j}\right)^{\alpha \beta}=\lambda_{i}^{(\alpha} \bar{\lambda}_{i}^{\beta)}+\lambda_{j}^{(\alpha} \bar{\lambda}_{j}^{\beta)} .
$$

In a supersymmetric theory, one also has supermomenta. Assuming the theory has $\mathscr{N}=2$ supersymetry, the sum of the supermomenta of legs $i$ and $j$ is given by

$$
\left(q_{i}+q_{j}\right)^{\alpha}=\lambda_{i}^{\alpha} \bar{\eta}_{i}+\bar{\lambda}_{i}^{\alpha} \eta_{i}+\lambda_{j}^{\alpha} \bar{\eta}_{j}+\bar{\lambda}_{j}^{\alpha} \eta_{j}
$$


It is not difficult to see that the supermomentum is conserved by the following deformation:

$$
\left\{\left(\begin{array}{c}
\lambda_{i} \\
\lambda_{j}
\end{array}\right),\left(\begin{array}{c}
\bar{\lambda}_{i} \\
\bar{\lambda}_{j}
\end{array}\right),\left(\begin{array}{c}
\eta_{i} \\
\eta_{j}
\end{array}\right),\left(\begin{array}{c}
\bar{\eta}_{i} \\
\bar{\eta}_{j}
\end{array}\right)\right\} \rightarrow\left\{R(z)\left(\begin{array}{c}
\lambda_{i} \\
\lambda_{j}
\end{array}\right), R(z)\left(\begin{array}{c}
\bar{\lambda}_{i} \\
\bar{\lambda}_{j}
\end{array}\right), R(z)\left(\begin{array}{c}
\eta_{i} \\
\eta_{j}
\end{array}\right), R(z)\left(\begin{array}{c}
\bar{\eta}_{i} \\
\bar{\eta}_{j}
\end{array}\right)\right\}
$$

where

$$
R(z)=\left(\begin{array}{cc}
\frac{1}{2}\left(z+z^{-1}\right) & \frac{i}{2}\left(z-z^{-1}\right) \\
-\frac{i}{2}\left(z-z^{-1}\right) & \frac{1}{2}\left(z+z^{-1}\right)
\end{array}\right) .
$$

Furthermore, if we choose phase of $\lambda_{i}$ or $\lambda_{j}$ such that $\langle i \bar{j}\rangle=\langle\bar{i} j\rangle$, then the deformation preserves $\langle i \bar{i}\rangle$ and $\langle j \bar{j}\rangle$. In massless limit, this deformation reduces to BCFW deformation for massless gauge theories proposed in [1]. Note that deformation becomes trivial when $z=1$.

After performing the deformation in equations 3.3 and 3.4, an on-shell amplitude $\mathscr{A}$ will develope poles in the complex parameter $z$. Near these poles, the amplitude will factorize into two on-shell amplitudes (denoted $\mathscr{A}_{L}$ and $\mathscr{A}_{R}$ ) multiplied by a propagator. Using this fact and assuming that the amplitude vanishes at $z \rightarrow \infty$, one finds that

$$
\mathscr{A}(z=1)=-\frac{1}{2 \pi i} \sum_{f, j} \int d \eta \oint_{z f, j} \frac{\mathscr{A}_{L}(z, \eta) \mathscr{A}_{R}(z, i \eta)}{\hat{p}_{f}(z)^{2}+m^{2}} \frac{1}{z-1}
$$

where the factorization channels are labeled by $f$, and $z_{f, j}$ corresponds to the $j$ th root of $\hat{p}_{f}(z)^{2}+$ $m^{2}$. In obtaining this formula, we assumed that all external legs of on-shell amplitudes have the same mass $m$. The integral $\int d \eta$ takes into account all the fields in the supermultiplet which can appear in the propagator. Using this equation, one can compute higher-point on-shell amplitudes from lower-point on-shell amplitudes. From the deformation in equations 3.3 and 3.4, one can see that in any channel, $\hat{p}_{f}(z)^{2}+m^{2}$ has the following form

$$
\hat{p}_{f}(z)^{2}+m^{2}=a_{f} z^{-2}+b_{f}+c_{f} z^{2}
$$

Hence the poles in each factorzation channel are obtained by solving a quadratic equation in $z^{2}$.

In order for the BCFW recursion relation to be applicable, the on-shell amplitudes must vanish after performing the deformation and taking the deformation parameter $z$ to infinity. Although the amplitudes of the Yang-Mills-Chern-Simons theory do not generally have good large- $z$ behavior, the 4-point superamplitude in eq 3.2 is $\mathscr{O}(1 / z)$ when legs $(1,3)$ are shifted, so the recursion relation may be applicable to the superamplitudes of the mass-deformed Chern-Simons theory. In order to test this, one should use the recursion relation to compute the 6-point superamplitude of the mass-deformed Chern-Simons theory, and match various components of the superamplitude with Feynman diagram calculations.

\section{Conclusion}

In this note we have reviewed recent progress in understanding the planar, on-shell, colorordered scattering amplitudes of various $3 \mathrm{~d}$ gauge theories, notably maximal $3 \mathrm{~d}$ super-Yang-Mills 
theory, $\mathscr{N}=2$ mass-deformed Chern-Simons theory, and $\mathscr{N}=2$ Yang-Mills-Chern-Simons theory. Although 3d Yang-Mills theories can be obtained by dimensional reduction of $4 \mathrm{~d}$ Yang-Mills theories, their amplitudes exhibit many intersting properties which do not follow trivially from dimensional reduction. For example, the amplitudes of maximal 3d super-Yang-Mills theory exhibit enhanced R-symmetry and dual conformal covariance. Furthermore, the loop amplitudes of this theory have a similar structure to those of the ABJM theory, which is an $\mathscr{N}=6$ superconformal Chern-Simons theory. The scattering amplitudes of mass deformed Chern-Simons theory and Yang-Mills-Chern-Simons theory also exhibit a number of interesting properties. For example, the four point amplitudes of the mass-deformed $\mathscr{N}=2$ Chern-Simons theory can be encoded in a very simple superamplitude, eq 3.2. In the Yang-Mills-Chern-Simons theory, the gauge field has one massive propagating degree of freedom, and can therefore appear in the external legs of on-shell amplitudes. In particular, the Chern-Simons term gives rise to a topological mass for the gauge field. Although there are difficulties associated with computing amplitudes with external gauge fields in Yang-Mills-Chern-Simons theories using Feynman diagrams, it is possible to compute amplitudes without external gauge fields and then deduce the remaining amplitudes using supersymmetry, at least at four-points in the $\mathscr{N}=2$ Yang-Mills-Chern-Simons theory.

There are a number of open questions. For example, it would be interesting to study the loop amplitudes of mass-deformed $\mathscr{N}=2$ gauge theories in three dimensions. It would also be interesting to apply the techniques described in this note to study the scattering amplitudes of $3 \mathrm{~d}$ gauge theories with spontaneously broken gauge symmetry. In this case, masses are acquired via the Higgs mechanism.

\section{References}

[1] D. Gang, Y. -t. Huang, E. Koh, S. Lee and A. E. Lipstein, "Tree-level Recursion Relation and Dual Superconformal Symmetry of the ABJM Theory," JHEP 1103, 116 (2011) [arXiv:1012.5032 [hep-th]].

[2] O. Aharony, O. Bergman, D. L. Jafferis and J. Maldacena, " $\mathrm{N}=6$ superconformal Chern-Simons-matter theories, M2-branes and their gravity duals," JHEP 0810, 091 (2008) [arXiv:0806.1218 [hep-th]].

[3] T. Bargheer, F. Loebbert and C. Meneghelli, "Symmetries of Tree-level Scattering Amplitudes in N=6 Superconformal Chern-Simons Theory,” Phys. Rev. D 82, 045016 (2010) [arXiv:1003.6120 [hep-th]].

[4] Y. -t. Huang and A. E. Lipstein, "Dual Superconformal Symmetry of N=6 Chern-Simons Theory," JHEP 1011, 076 (2010) [arXiv:1008.0041 [hep-th]].

[5] J. M. Henn, J. Plefka and K. Wiegandt, "Light-like polygonal Wilson loops in 3d Chern-Simons and ABJM theory," JHEP 1008, 032 (2010) [Erratum-ibid. 1111, 053 (2011)] [arXiv:1004.0226 [hep-th]].

[6] W. -M. Chen and Y. -t. Huang, "Dualities for Loop Amplitudes of N=6 Chern-Simons Matter Theory," JHEP 1111, 057 (2011) [arXiv:1107.2710 [hep-th]].

[7] M. S. Bianchi, M. Leoni, A. Mauri, S. Penati and A. Santambrogio, "Scattering Amplitudes/Wilson Loop Duality In ABJM Theory,” JHEP 1201, 056 (2012) [arXiv:1107.3139 [hep-th]].

[8] S. Lee, "Yangian Invariant Scattering Amplitudes in Supersymmetric Chern-Simons Theory," Phys. Rev. Lett. 105, 151603 (2010) [arXiv:1007.4772 [hep-th]]. 
[9] M. S. Bianchi, M. Leoni, A. Mauri, S. Penati and A. Santambrogio, "One Loop Amplitudes In ABJM," arXiv:1204.4407 [hep-th].

[10] T. Bargheer, N. Beisert, F. Loebbert and T. McLoughlin, "Conformal Anomaly for Amplitudes in N=6 Superconformal Chern-Simons Theory,” arXiv:1204.4406 [hep-th].

[11] A. Brandhuber, G. Travaglini and C. Wen, "A note on amplitudes in N=6 superconformal Chern-Simons theory," arXiv:1205.6705 [hep-th].

[12] A. E. Lipstein and L. Mason, "Amplitudes of 3d Yang Mills Theory,” arXiv:1207.6176 [hep-th].

[13] A. Agarwal, A. E. Lipstein and D. Young, "Scattering Amplitudes of Massive N=2 Gauge Theories in Three Dimensions," arXiv:1302.5288 [hep-th].

[14] A. Agarwal and D. Young, "Manifest SO(N) invariance and S-matrices of three-dimensional N=2,4,8 SYM,” JHEP 1105, 100 (2011) [arXiv:1103.0786 [hep-th]].

[15] M. S. Bianchi and M. Leoni, "N=8 SYM vs. N=6 Chern-Simons: Four-point amplitudes at two-loops," arXiv:1210.4925 [hep-th].

[16] L. Brink, J. H. Schwarz and J. Scherk, “Supersymmetric Yang-Mills Theories,” Nucl. Phys. B 121, 77 (1977).

[17] D. -W. Chiou, O. J. Ganor, Y. P. Hong, B. S. Kim and I. Mitra, "Massless and massive three dimensional super Yang-Mills theory and mini-twistor string theory," Phys. Rev. D 71, 125016 (2005) [hep-th/0502076].

[18] A. Agarwal, N. Beisert and T. McLoughlin, "Scattering in Mass-Deformed N>=4 Chern-Simons Models,” JHEP 0906, 045 (2009) [arXiv:0812.3367 [hep-th]].

[19] Z. Bern, L. J. Dixon, D. C. Dunbar and D. A. Kosower, "One loop n point gauge theory amplitudes, unitarity and collinear limits,” Nucl. Phys. B 425, 217 (1994) [hep-ph/9403226].

[20] S. Deser, R. Jackiw and S. Templeton, "Topologically Massive Gauge Theories," Annals Phys. 140, 372 (1982) [Erratum-ibid. 185, 406 (1988)] [Annals Phys. 185, 406 (1988)] [Annals Phys. 281, 409 (2000)].

[21] S. Deser, R. Jackiw and S. Templeton, "Three-Dimensional Massive Gauge Theories,” Phys. Rev. Lett. 48, 975 (1982).

[22] R. Britto, F. Cachazo, B. Feng and E. Witten, "Direct proof of tree-level recursion relation in Yang-Mills theory," Phys. Rev. Lett. 94, 181602 (2005) [hep-th/0501052]. 